\title{
Anatomia cardíaca e ramificações da aorta em macaco-prego (Sapajus apella)
}

\author{
Daniely Figueiró Silva Furtado \\ Luanna Dienyfer Prata Vasconcelos \\ Érika Branco \\ Ana Rita de Lima * \\ Universidade Federal Rural da Amazônia, Faculdade de Medicina Veterinária \\ Instituto da Saúde e Produção Animal, Laboratório de Pesquisa Morfológica Animal \\ Avenida Presidente Tancredo Neves, 2501, CEP 66.077-530, Belém - PA, Brasil \\ * Autor para correspondência \\ vetlima@uol.com.br
}

Submetido em 24/03/2017

Aceito para publicação em 02/08/2017

\section{Resumo}

O objetivo do presente trabalho foi descrever a anatomia cardíaca da espécie Sapajus apella, fazendo um estudo da morfologia e irrigação cardíaca, aorta abdominal e suas ramificações em duas fêmeas e dois machos desta espécie. Os animais foram dissecados em nível de arco aórtico, o qual foi canulado e nele injetado solução de látex Neoprene 650, corado com pigmento vermelho. Em seguida, foram fixados em solução aquosa de formoldeído a 10\%. O coração dos exemplares analisados apresentava-se perfeitamente envolto pelo pericárdio, com a base do órgão na altura da terceira costela e localização dorsal. A morfologia interna do coração e suas ramificações revelam grande parte de suas estruturas-padrões descritas na literatura. As ramificações da aorta abdominal mostraram-se semelhantes às de outras espécies já descritas na literatura, apresentando como primeiro ramo a artéria celíaca, que se trifurcou de maneira clássica em gástrica esquerda, esplênica e hepática. O ramo seguinte foi o da artéria mesentérica cranial, seguido das artérias renais e mesentérica caudal. A região terminal da aorta originou as ilíacas externas e internas e, por fim, a sacral mediana, que estava ausente em um dos animais analisados.

Palavras-chave: Primata; Ramos Colaterais; Sistema Cardiovascular

\section{Abstract}

Cardiac anatomy and aortic ramifications in the capuchin monkey (Sapajus apella). The objective of this study was to describe the anatomy of the heart of Sapajus paella. The cardiac morphology, irrigation, and the abdominal aorta and its branches were studied in two females and two males. The animals were dissected at the level of the aortic arch, which was cannulated, injected with a solution of neoprene latex 650 , stained with red pigment and fixed in an aqueous solution of $10 \%$ formaldehyde. The heart of the analyzed animals was perfectly wrapped by the pericardium, with the base of the organ at the third rib and dorsal side. The internal morphology of the heart and its ramifications revealed a lot of the structural patterns described in the literature. The branches of the abdominal aorta were similar to those of other described species. The first branch was the 
celiac artery that classically trifurcated into the left gastric, splenic and liver arteries. The next branch was the cranial mesenteric artery, followed by the kidney arteries and caudal mesenteric artery. The terminal region of the aorta originated in the external and internal iliac arteries and, finally, the median sacral artery, which was absent in one of the animals analyzed.

Key words: Cardiovascular System; Collateral Branches; Primates

\section{Introdução}

A espécie Sapajus apella é conhecida por sua grande tolerância de sobrevivência em ambientes alterados pelo homem (LUDWIG et al., 2005), sendo, por isso, uma espécie sem risco de extinção (IUCN, 2013).

De acordo com Dyce et al. (2010), o coração é o órgão que realiza o bombeamento de sangue continuamente por meio dos vasos sanguíneos. Em mamíferos, apresenta quatro câmaras que contraem ritmicamente, sendo dois átrios e dois ventrículos. Os átrios direito e esquerdo são separados pelo septo interatrial, e os ventrículos direito e esquerdo, pelo septo interventricular.

A aorta abdominal é a porção da aorta descendente que penetra na cavidade abdominal após atravessar o hiato aórtico do diafragma. Acompanha o teto do abdômen, relacionando-se com a veia cava caudal a sua direita e com o músculo psoas a sua esquerda. É responsável pela vascularização de todos os órgãos da cavidade abdominal e pélvica, além dos membros pélvicos (DYCE et al., 2010).

Estudos referentes à morfologia cardíaca têm levado esta linha de pesquisa a um patamar mais avançado, principalmente pelo desenvolvimento de estudos de natureza comparativa (BIASI, 2009). Assim, objetivou-se a descrição da morfologia e da irrigação cardíaca, além das ramificações da aorta desta espécie.

\section{Material e Métodos}

Foram utilizados quatro animais adultos da espécie Sapajus apella, sendo duas fêmeas e dois machos, provenientes do Centro Nacional de Primatas, sob aprovação do Comitê de Pesquisa com Animais (CEPAN/IEC/SVS/MS) no 008/2010, doados após óbito por causas naturais ao Laboratório de Pesquisa Morfológica Animal (LaPMA) da Universidade Federal Rural da Amazônia (UFRA). Os animais foram dissecados em nível de arco aórtico (através do $5^{\circ}$ espaço intercostal esquerdo), o qual foi canulado e nele injetado solução de látex Neoprene 650, corado com pigmento vermelho. Em seguida, os animais foram fixados em solução aquosa de formoldeído a $10 \%$ por perfusão intramuscular, subcutânea e intracavitária, com posterior submersão dos cadáveres na mesma solução fixadora, por um período mínimo de sete dias.

Após o processo de fixação, foi feita uma incisão desde a região mentoniana até a região pélvica, seguida pelo rebatimento de pele e musculatura. Foi observada e posteriormente descrita a exata localização do coração de cada exemplar. O arco aórtico dos espécimes também foi dissecado, identificado e analisado, assim como a aorta abdominal e a origem de seus ramos colaterais. Após a realização de registros fotográficos e a identificação das artérias de interesse neste estudo, o coração foi individualizado com o uso de bisturi, pinças e tesouras cirúrgicas. As artérias coronárias e suas colaterais foram dissecadas e identificadas e foram feitas análises e fotografias das cavidades cardíacas tanto interna quanto externamente. Toda nomenclatura adotada foi baseada na Nomina Anatômica Veterinária (INTERNATIONAL COMMITTEE ON VETERINARY GROSS ANATOMICAL NOMENCLATURE, 2012).

\section{Resultados}

Cada coração analisado apresentava-se perfeitamente envolto pelo pericárdio, com localização assimétrica devido à sua inclinação para o lado esquerdo do plano mediano, mantendo-se estabilizado no local devido à presença de uma estrutura que liga o pericárdico ao diafragma - o ligamento freno pericárdico (Figura 1). 
Os quatro animais estudados apresentaram a base do coração na altura da terceira costela, com localização dorsal. O ápice foi observado na altura da sétima costela, dorsal ao esterno.

Os corações dos exemplares estudados foram analisados externa e internamente, apresentando forma de cone irregular, com presença de sulcos que dividem o órgão em faces e evidenciam a organização do órgão em quatro câmaras: átrios direito e esquerdo e ventrículos direito e esquerdo. Quando da retirada do pericárdio, o órgão apresentava-se íntegro, com a presença do epicárdio envolvendo-o por completo (Figura 2). Não se notou a presença de gordura pericárdica, epicárdica ou subepicárdica em nenhum dos espécimes estudados. Sob o epicárdio pode-se observar o miocárdio.

FIGURA 1: Fotomacrografia demonstrando a localização in situ do coração, onde é observada a inclinação cardíaca para o lado esquerdo do tórax. Podemos visualizar o ventrículo esquerdo (1), o ventrículo direito (2), a origem do arco aórtico (3), o lobo pulmonar direito (4) e parte do diafragma (5). Barra de escala: $1,0 \mathrm{~cm}$.

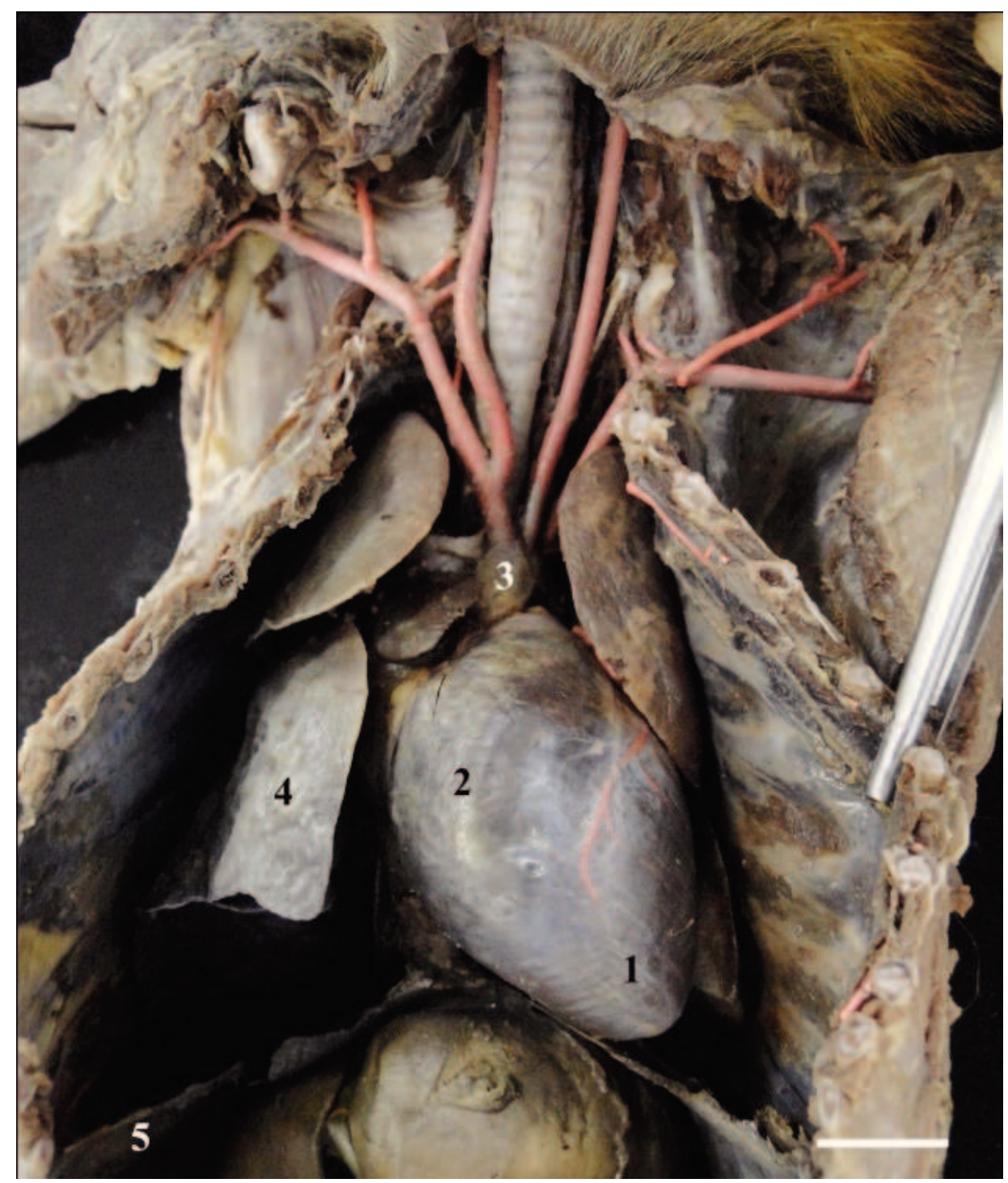


FIGURA 2: Fotomacrografias demonstrando o posicionamento do coração in situ na cavidade torácica. Em A, pode-se visualizar a presença do pericárdio envolvendo o coração (1) além do pulmão direito (2) e da origem do arco aórtico (3). Em B, visualiza-se o coração com o pericárdio removido (1), o pulmão direito rebatido (2) e a origem do arco aórtico (3). Barra de escala: $1,0 \mathrm{~cm}$.

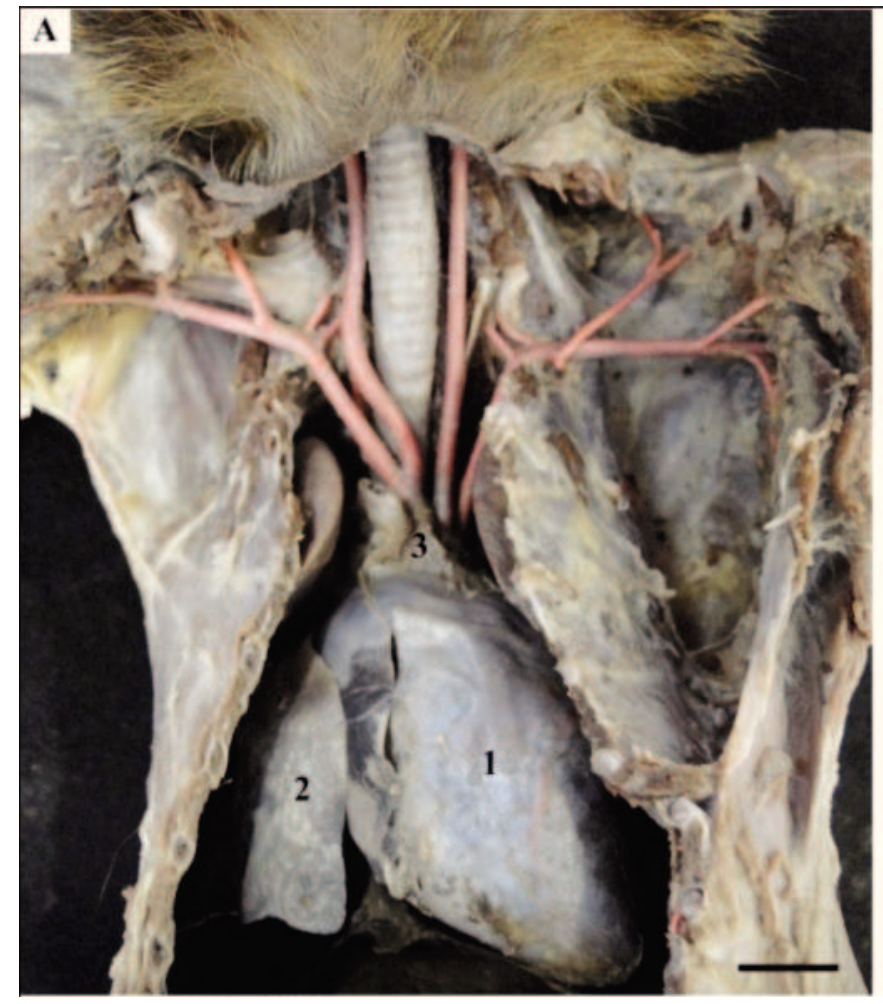

O arco aórtico, logo após a sua origem, apresenta uma curvatura à esquerda e segue caudalmente, sendo assim chamado de aorta torácica. Do arco aórtico surge o tronco braquiocefálico, de onde partem a artéria carótida comum direita e a subclávia direita; a artéria carótida comum esquerda e a artéria subclávia esquerda (Figura 3).

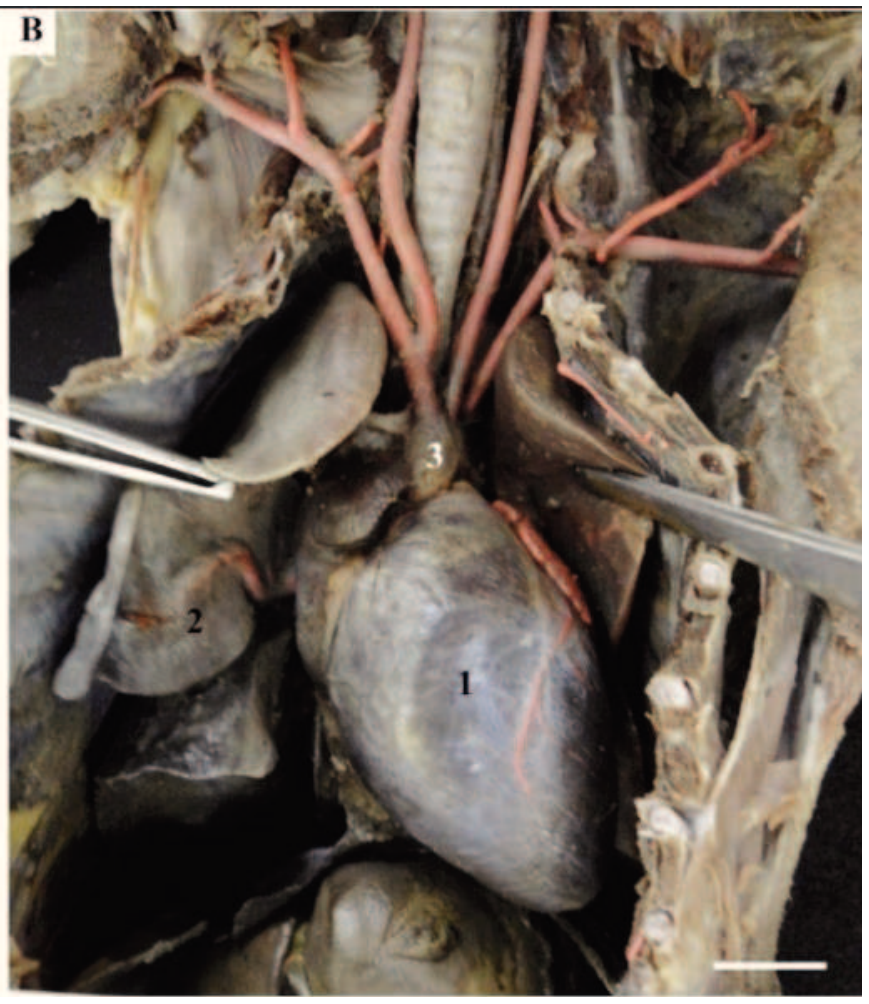

Na análise ex situ, identificaram-se alguns dos vasos mais importantes constituintes do sistema de irrigação do músculo cardíaco. Na vista anterior observa-se a presença do ramo interventricular paraconal da artéria coronária esquerda, o qual tem sua terminação próxima ao ápice do coração. Notam-se também ramos diagonais partindo dele e, na sua vista posterior, identificou-se o ramo posterior do ventrículo esquerdo (Figura 4). 
FIGURA 3: Fotomacrografia evidenciando os três vasos originários do arco aórtico e suas principais ramificações. Em A, é possível observar o arco aórtico (1), tronco braquiocefálico (2), artéria subclávia direita (3), artéria carótida comum direita (4), artéria carótida comum esquerda (5), artéria subclávia esquerda (6), artéria vertebral direita (7) e esquerda (8), artéria axilar direita (9) e esquerda (10), artéria torácica interna direita (11) e esquerda (12). Em B, observa-se o arco aórtico (1), tronco braquiocefálico (2), artéria subclávia direita (3), artéria carótida comum direita (4), artéria carótida comum esquerda (5) e artéria subclávia esquerda (6). Barra de escala: $1,0 \mathrm{~cm}$.
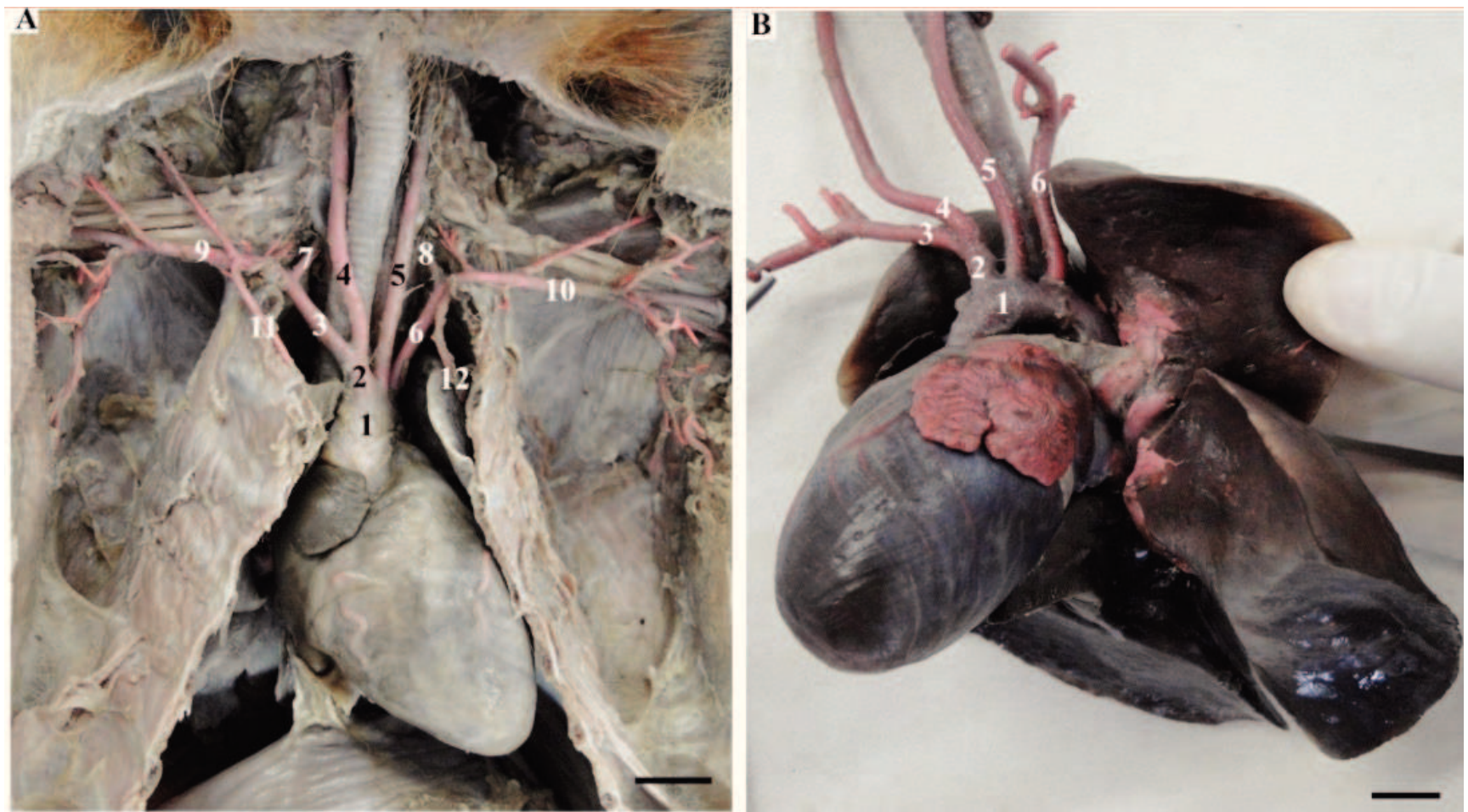

FIGURA 4: Fotomacrografias demonstrando a localização das artérias responsáveis pela irrigação do músculo cardíaco. Em A, observamse o ramo interventricular anterior da artéria coronária esquerda (1) e também os ramos diagonais do ramo interventricular anterior da artéria coronária esquerda (2). Barra de escala: $1,5 \mathrm{~cm}$. Em B, nota-se em destaque o ramo posterior do ventrículo esquerdo (3). Barra de escala: $1,0 \mathrm{~cm}$.
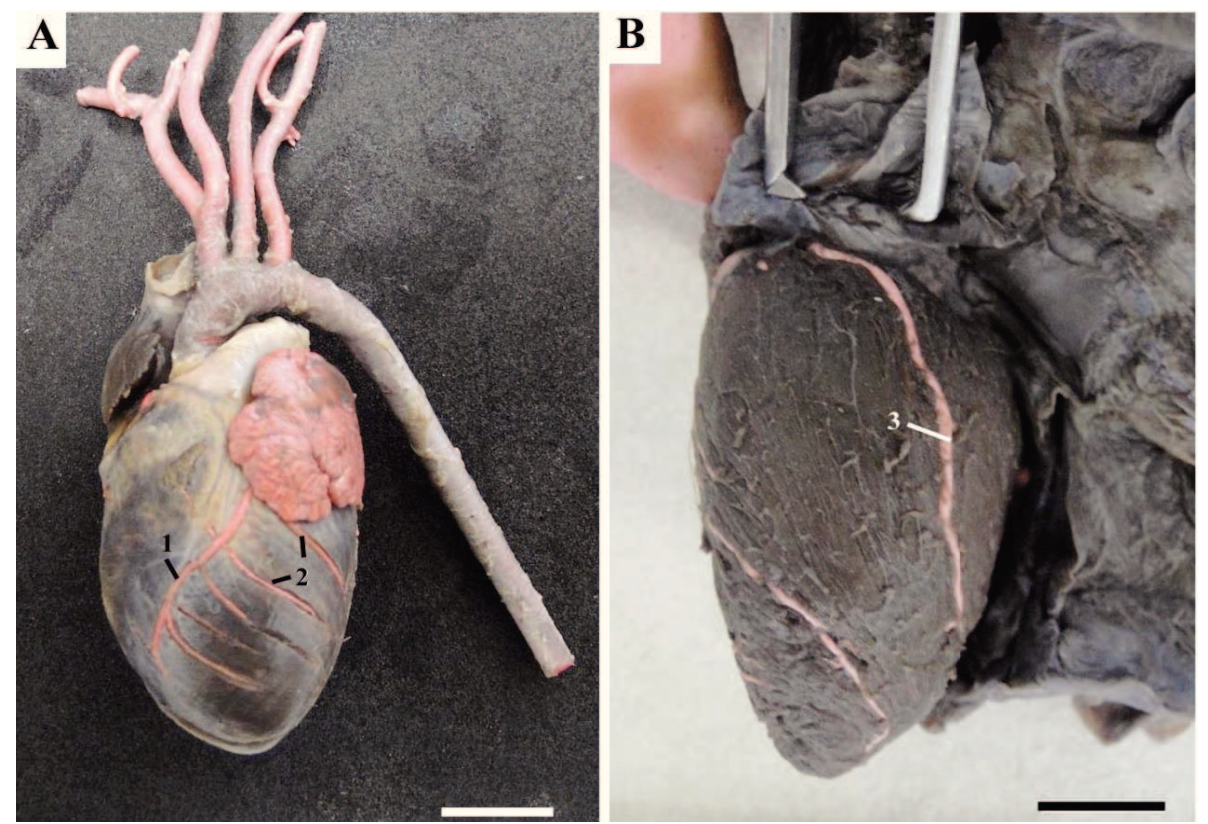
A morfologia interna do coração revela grande parte de suas estruturas-padrões. Nota-se a presença dos dois ventrículos cardíacos, além do septo que os separao septo interventricular. No ventrículo esquerdo, a valva aórtica, constituída por três válvulas semilunares, pode ser identificada claramente, assim como a valva mitral, também chamada de valva bicúspide ou atrioventricular esquerda. Ainda foi possível identificar as cordas tendíneas, trabéculas carnosas e músculo papilar (Figura 5). No ventrículo direito, foi possível identificar a valva pulmonar, constituída por três válvulas semilunares, a valva tricúspide ou atrioventricular direita, além dos músculos papilares e cordas tendíneas.

A aorta abdominal do $S$. apella apresentou origem na décima primeira vértebra torácica (T11), emergindo na cavidade abdominal após atravessar o diafragma na região do hiato aórtico. A primeira ramificação visceral, originada caudalmente ao diafragma e ventralmente à aorta abdominal, foi a artéria celíaca, artéria ímpar e curta que, logo em seguida, classicamente se trifurcou emitindo as artérias hepática, gástrica esquerda e esplênica, vascularizando fígado, estômago e baço respectivamente (Figuras 6A e 6B).

A artéria gástrica esquerda apresentou uma projeção em direção à cárdia, emitindo ramos esofágicos e liberando ramos para as superfícies parietal e visceral do estômago. A artéria hepática, a mais calibrosa das três, dirigiu-se para o fígado, vascularizando também o estômago, pâncreas e duodeno. A artéria esplênica irrigou o baço, o pâncreas e a região cárdica do estômago (Figuras 6A e 6B).

Ventrolateralmente à artéria celíaca, surgiram as artérias adrenais direita e esquerda. Quanto à origem dessas artérias, restringiu-se somente à participação da aorta abdominal (Figuras 6A e 6B).

FIGURA 5: Fotomacrografias evidenciando as estruturas internas cardíacas. Em A, nota-se o septo interventricular (1) separando o ventrículo esquerdo (2) do ventrículo direito (3). Observa-se claramente a presença da valva aórtica (4) e mitral (5), além do Músculo papilar (*) e de trabéculas carnosas (seta branca). Em B, sob outro ângulo de corte, notam-se o ventrículo esquerdo (1), valva aórtica (2) e mitral (3), além do músculo papilar (4), cordas tendíneas (setas brancas) e trabécula carnosa (seta preta). Barra de escala: $1,5 \mathrm{~cm}$.
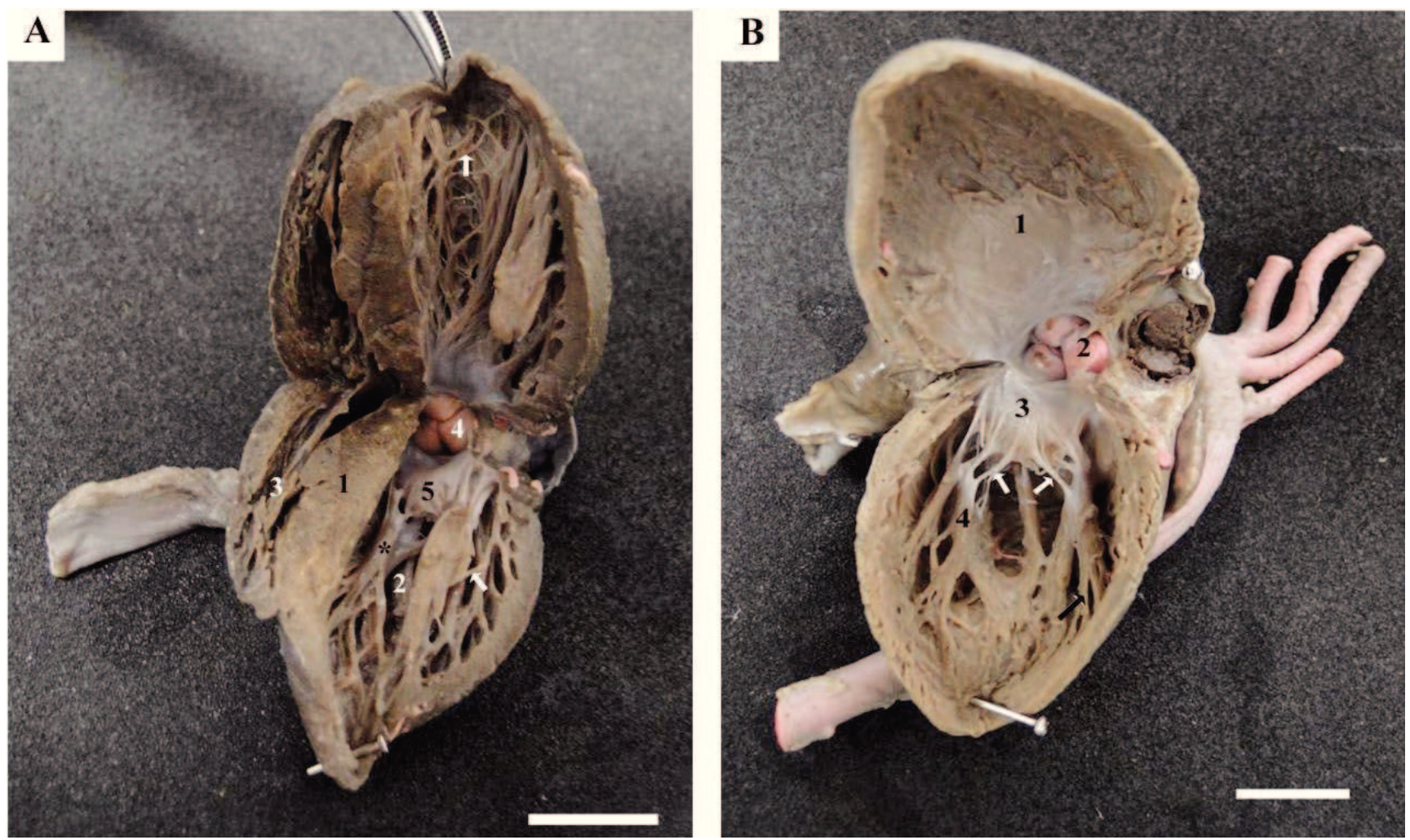
FIGURA 6: Aorta abdominal e seus ramos colaterais viscerais em Sapajus apella. Em A: a. celíaca (1), a. hepática (2), fígado (3), a. gástrica esquerda (4), a. esplênica (5), baço (6), a. mesentérica cranial (7), Aa. Renais direita e esquerda (8 e 8'), rins direito e esquerdo (9 e 9'), aorta abdominal (10), Aa. Testiculares direita e esquerda (11 e 11'), a. mesentérica caudal (12), Aa. Ilíacas externas direita e esquerda (13 e 13'), Aa. Ilíacas internas direita e esquerda (14 e 14'), Aa. Circunflexas ilíacas profundas direita e esquerda (15 e 15') e A. adrenal esquerda (16). Em B: Desenho esquemático em vista ventral da aorta abdominal e seus principais ramos colaterais viscerais. Barra de escala: $1 \mathrm{~cm}$.
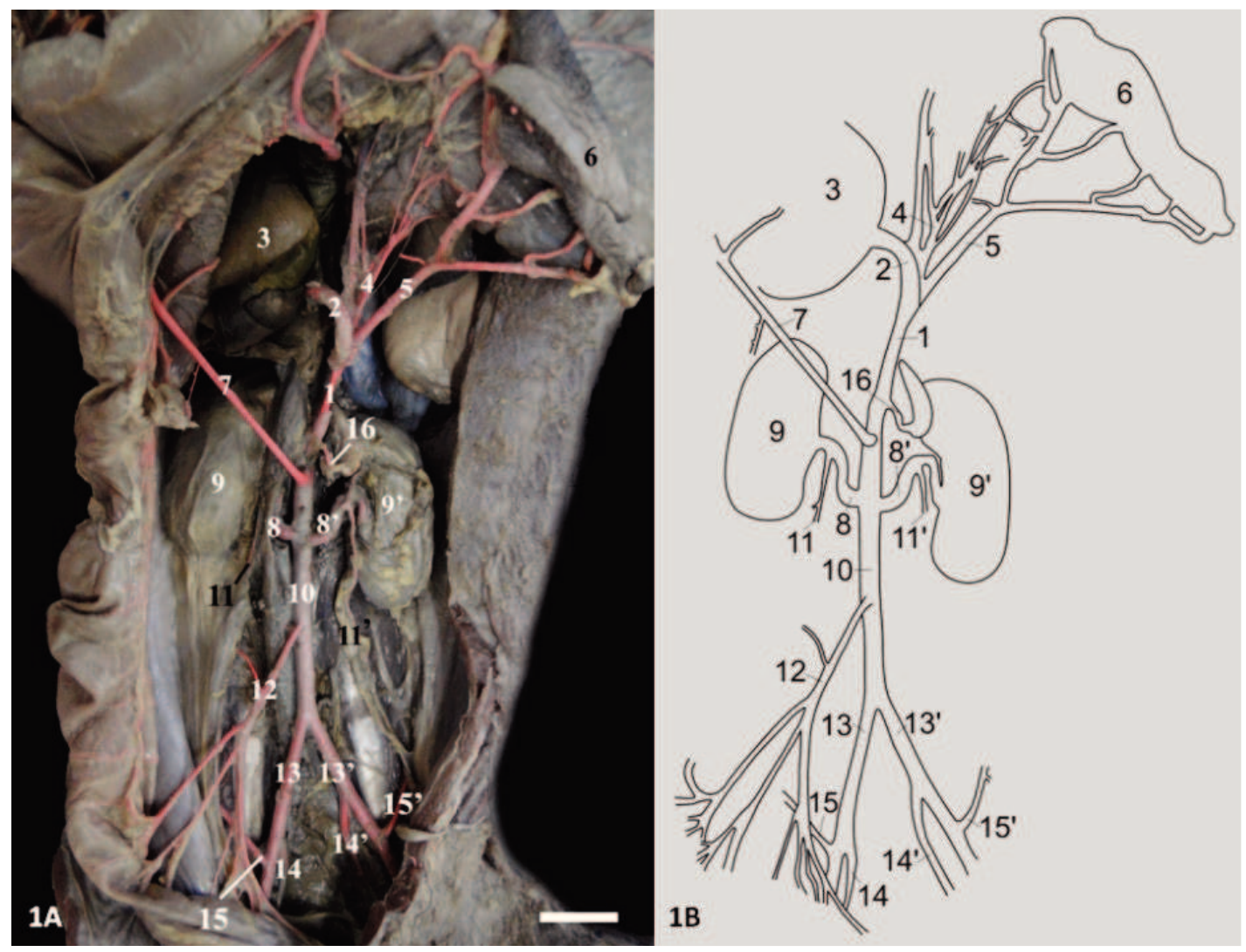

O ramo originário seguinte da face ventral da aorta abdominal foi a artéria mesentérica cranial. Logo após sua origem, penetrou no mesentério irrigando principalmente a parte caudal do duodeno até a parte cranial do cólon descendente (Figuras 6A e 6B).

Após a artéria mesentérica cranial, surgiram dois ramos colaterais chamados de artérias renais, que tiveram origem assimétrica acompanhando a topografia dos rins. As artérias testiculares se originaram da porção terminal das artérias renais, cada uma delas seguiu lateralmente no sentido do anel inguinal, tornando-se constituinte do funículo espermático (Figuras 6A e 6B).

Caudalmente as artérias renais, teve origem a artéria mesentérica caudal, que supriu a região terminal do cólon descendente e a região cranial do reto (Figuras $6 \mathrm{~A}$ e $6 \mathrm{~B})$.
A região terminal da aorta abdominal se bifurcou e dela se derivaram os ramos das artérias ilíacas externas direita e esquerda; estas, por sua vez, bifurcam-se em artérias ilíacas internas direita e esquerda. A continuidade da aorta abdominal, após a bifurcação, em artérias ilíacas externas deu origem à artéria sacral mediana (Figura 7). Dos quatro animais estudados, três apresentaram a conformação clássica da porção terminal da aorta descrita no parágrafo anterior, sendo que em um desses animais observou-se uma variação anatômica representada pela ausência da artéria sacral mediana (Figura 6A). Das artérias ilíacas externas, originaram-se as artérias circunflexas ilíacas profundas direita e esquerda, que irrigam os músculos da região abdominal (Figuras 6A, 6B e 7). 
FIGURA 7: Porção terminal da aorta abdominal de três dos quatro animais da espécie Sapajus apella. Notaramse porção terminal da aorta (1), Aa. Ilíacas externas direita e esquerda ( 2 e 2 '), Aa. Ilíacas internas direita e esquerda (3 e 3'), a. sacral mediana (4) e Aa. Circunflexas ilíacas profundas direita e esquerda (5 e 5'). Barra de escala: $1 \mathrm{~cm}$.

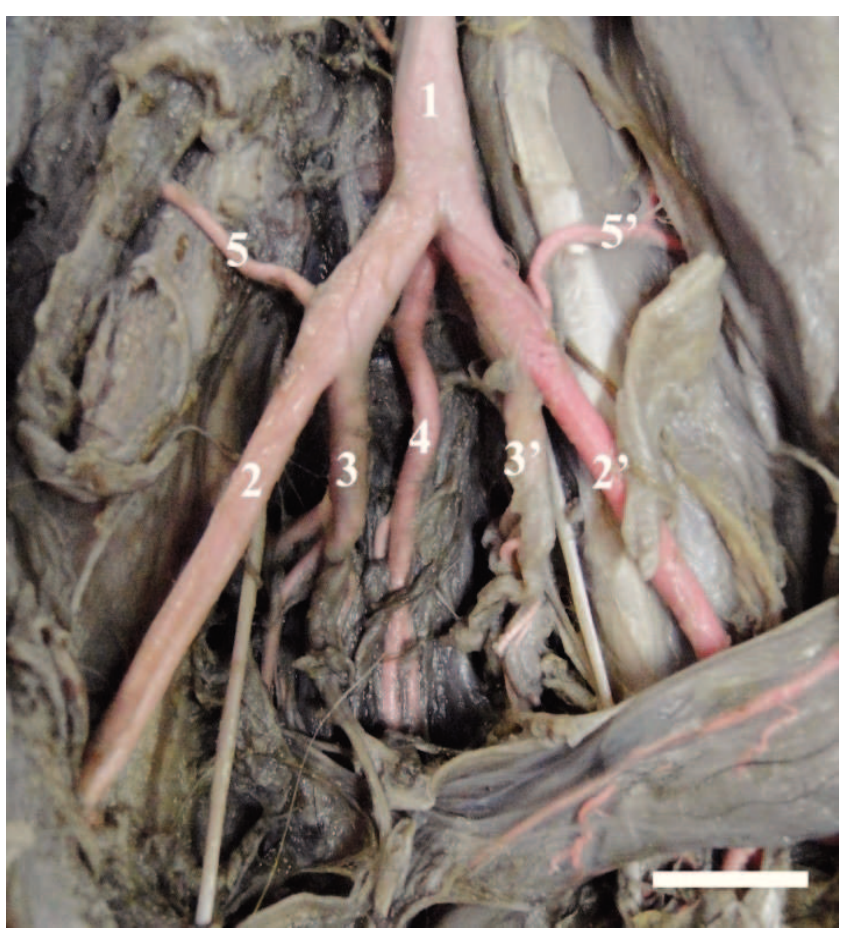

\section{Discussão}

A localização do órgão, assim como a presença do saco pericárdico no Sapajus apella, é semelhante ao que foi descrito para o Saguinus niger (PINTO et al., 2011). No caso do Sapajus apella, o órgão se mantinha estabilizado no local devido à presença de uma estrutura que liga o saco pericárdico ao diafragma - o ligamento freno pericárdico, como também foi observado nos estudos de Binvignat e Olave (2009) no homem (Homo sapiens sapiens). Assim como encontrado em avestruzes, em estudo desenvolvido por Soares et al. (2010).

Com relação à morfologia cardíaca interna, grande parte das estruturas encontradas rotineiramente em mamíferos estava presente, sendo a descrição no presente estudo bastante semelhante àquela definida por Pinto et al. (2011) em achados na espécie Saguinus niger. Contudo, há estudos envolvendo diferentes espécies mamíferos em que é observada a presença da valva quadricúspide, uma anomalia congênita rara. López Garcia et al. (2015), realizando experimentos com hamsters-sírios (Mesocricetus auratus), e Michlik et al. (2014), em um relato de caso com cavalo doméstico, descreveram tal alteração.

Os dados relacionados ao surgimento do tronco braquiocefálico e suas ramificações em subclávias (esquerda e direita) e carótida comum esquerda coincidem com as descrições em estudos que tratavam do padrão de ramificação dos vasos do arco aórtico em seres humanos no Sul da Índia e no Peru (NAYAK et al., 2006; REKHA; SENTHILKUMAR, 2013; HUAPAYA et al., 2015). Entretanto, Pinheiro et al. (2014) descreveram que no Tamanduá-mirim (Tamandua tetradactyla) o padrão de ramificação é diferente: o tronco braquiocefálico dá origem à artéria subclávia direita, com a esquerda partindo diretamente do arco aórtico.

A terminação da artéria coronária esquerda próxima ao ápice do coração corrobora o que foi encontrado por McNamara et al. (1973), em um estudo sobre variações na forma de distribuição dessa artéria em humanos, podendo o Sapajus apella ser enquadrado no tipo I, no qual a porção final desse ramo está localizada no ápice do coração, ou próxima a ele. Observa-se que cinco ramos diagonais têm sua origem no ramo interventricular da artéria coronária esquerda, descrição essa não encontrada no estudo de Ortale et al. (2005) com anatomia dos ramos vasculares em coração humano.

Assim como na maioria dos animais domésticos (DYCE et al., 2004) e silvestres, como as cutias (Dasyprocta aguti) (CARVALHO et al., 1999), a artéria celíaca do Sapajus apella é a primeira ramificação da aorta abdominal. Sua origem, na face ventral da aorta e imediatamente caudal ao hiato aórtico, ocorreu separadamente da artéria mesentérica cranial, como em $70 \%$ das 10 nutrias (Myocastor coypus) estudadas por Machado et al. (2002) e em suínos, coelhos e cães. Já nos ovinos, a origem da artéria celíaca ocorre por tronco comum (GHOSHAL, 1986; EVANS, 1993).

Ainda, ao que se refere à origem da artéria celíaca na face ventral da aorta, vários autores descreveram esse comportamento na artéria celíaca em diferentes mamíferos (KENNEDY; SMITH, 1930; BERG, 1961; 
SLEIGHT; THOMFORD, 1970; ENGE; FLATMARK, 1972; SCHMIDT et al., 1980; NICKEL et al., 1983; BEDNAROVA; MALINOVSKI, 1984; NIZA et al., 2003; ABIDU-FIGUEIREDO et al., 2005; 2008). Entretanto, em estudo realizado com fetos de bubalinos, Machado et al. (2000) relataram a origem da artéria celíaca ocorrendo ao nível da porção torácica da aorta, discordando com os presentes resultados.

Corroborando os achados de Ghoshal (1986) e Evans (1993), em cão, com Cavalcante Filho et al. (1998), nos queixadas, Bonatelli et al. (2003), em tamanduá-bandeira, Culau et al. (2008), em nutria, e Macedo et al. (2013), em tamanduá-mirim, a artéria celíaca do $S$. apella apresentou-se ímpar e curta, dividindo-se nas artérias gástrica esquerda, hepática e esplênica. Essa descrição difere do observado em gatos, que apresentam dois arranjos, ou seja, a trifurcação clássica (como a do presente estudo) e uma bifurcação onde a artéria celíaca é formada pela artéria hepática e pelo tronco gastrolienal, que é a origem comum da artéria gástrica esquerda e esplênica (BEDNAROVA; MALINOVSKI, 1984; XAVIER-SILVA et al., 2012).

Com relação à artéria adrenal, Severino et al. (1992) relataram que em gatos S.R.D. a origem pode ocorrer de 1 a 4 ramos da aorta abdominal, 1 a 3 ramos da aorta abdominal, a 1 ou 2 ramos da artéria renal esquerda, a 1 ramo da artéria frênica caudal e a 2 ramos da artéria frênico abdominal. Essa observação vai contra os achados em $S$. apella, no qual a origem restringiu-se à aorta abdominal. Diferindo, também, dos achados de Silva et al. (2011), em Saimiri sciureus, em que a artéria adrenal esquerda teve origem na artéria celíaca e a direita na artéria renal direita. Difere também de Santos et al. (2011), nos estudos em lobo marinho, em que as adrenais direita e esquerda tiveram origem da aorta abdominal, artéria celíaca, artérias renais e artéria lombar direita. Os estudos com caprinos da raça Saanen (Capra hircus) (SILVA et al., 2003) mostraram a diversidade de artérias que contribuem para a origem das artérias adrenais, ou seja, as artérias adrenais direita e esquerda surgiram cada uma em média de cinco artérias.

O próximo ramo emitido da aorta abdominal do $S$. apella foi a artéria mesentérica cranial, que diferiu da nutria (CULAU et al., 2008), pois nesta ela surgiu bem próxima e caudalmente à artéria celíaca, sendo que no $S$. apella surgiu caudalmente às artérias adrenais. Assim como nos animais domésticos (DYCE et al., 2004), em alguns animais silvestres, como o ouriçocacheiro (Sphiggurus spp.) (MACHADO; ZIEMMAN, 2000), o ratão-do-banhado (Myocastor coypus) (MACHADO et al., 2006) e o rato de laboratório (HEBEL; STROMBERG, 1986), a artéria mesentérica cranial possui origem na face ventral da aorta abdominal. Ela supre a parte caudal do duodeno até a parte cranial do cólon descendente assim como em carnívoros domésticos (GHOSHAL, 1986).

Seguindo-se à artéria mesentérica cranial, surgiram as artérias renais direita e esquerda. Assim como no $S$. sciureus (SILVA et al., 2011), apresentaram-se como vasos calibrosos. Da mesma forma que é descrita nos cães (GHOSHAL, 1986), a artéria renal direita do $S$. apella surge um pouco mais cranial que a esquerda, em conformidade com a posição anatômica mais cranial do rim direito.

A artéria mesentérica caudal do Sapajus apella corroborou anatomicamente os modelos descritos em animais domésticos, sendo ímpar e surgindo na face ventral da aorta abdominal (GHOSHAL, 1986). Porém, diferiu dos achados de Carvalho et al. (1999) em cutias adultas, em que esta se bifurcava em artéria cólica esquerda e retal cranial.

As artérias testiculares do $S$. apella são emitidas como ramos colaterais das artérias renais, corroborando os achados em tamanduá-bandeira (BONATELLI et al., 2003) e em tamanduá-mirim (MACEDO et al., 2013), sendo classificadas como uma conformação atípica, pois, na literatura para animais domésticos (GHOSHAL, 1986) e para macaco Rhesus (GINTHER et al., 1974), as artérias gonadais são pequenos vasos que se originam ventrolateralmente da aorta abdominal. Por sua vez, as artérias ovarianas (a. ovarica) depois que se separam da aorta, seguem um curso contorcido até o ovário. Eles irrigam o ovário (ramus ovaricus) e destacam ramos para a tuba uterina e para o corno uterino (ramus uterinus). O ramo uterino forma anastomose com a artéria uterina no ligamento largo em mamíferos domésticos (KÖNIG; LIEBICH, 2011). 
Os ramos terminais da aorta abdominal do S. apella corroboram com os modelos já estudados para animais domésticos (DYCE et al., 2004). Assim como no Saimiri sciureus (SILVA et al., 2011), a artéria ilíaca externa bifurcou-se, dando origem à artéria ilíaca interna, tanto do antímero esquerdo quanto do direito, tendo a sacral mediana como ramo terminal da aorta abdominal. No gambá, a aorta abdominal termina como artérias ilíacas comuns, as quais emitem as artérias ilíacas internas e externas. No rato, as artérias ilíacas comuns dão origem à artéria ilíaca interna e continuam como artéria ilíaca externa, fato também observado na nutria, sendo a artéria sacral mediana, nesses animais, ramo da face dorsal da aorta abdominal, antes da divisão terminal (CULAU et al., 2008). Dos quatro animais estudados, um não apresentou a artéria sacral mediana, evidenciando uma variação anatômica.

As artérias circunflexas ilíacas profundas direita e esquerda no $S$. apella foram emitidas das artérias ilíacas externas direita e esquerda, corroborando os achados em nutria (FIGUEIREDO et al., 2008), diferentemente do que foi observado no coelho por Bavaresco et al. (2012), em que a artéria circunflexa ilíaca foi ramo da artéria ilíaca comum e em nenhum dos animais estudados foi constatada sua duplicidade.

\section{Referências}

ABIDU-FIGUEIREDO, M.; DIAS, G. P.; CERUTTI, S.; CARVALHO-DE-SOUZA, B.; MAIA, R. S.; BABINSKI, M. A. Variations of celiac artery in dogs: anatomic study for experimental, surgical and radiological practice. International Journal of Morphology, Temuco, v. 23, n. 1, p. 37-42, 2005.

ABIDU-FIGUEIREDO, M.; XAVIER-SILVA, B.; CARDINOT, T. M.; BABINSKI, M. A.; CHAGAS M. A. Celiac artery in New Zealand rabbit: anatomical study of its origin and arrangement for experimental research and surgical practice. Pesquisa Veterinária Brasileira, Seropédica, v. 28, n. 5, p. 237-240, 2008.

BAVARESCO, A. Z.; CULAU, P. O. V.; CAMPOS, R. Ramos colaterais parietais e terminais da aorta abdominal em coelhos da raça Nova Zelândia (Oryctolagus cuniculus). Acta Scientiae Veterinariae, Porto Alegre, v. 40, n. 4, p. 1-6, 2012.

BEDNAROVA, Z.; MALINOVSKY, L. Ramification of celiac artery in the domestic cat. Folia Morphologica, Gdańsk, v. 34, n. 1, p. 36-44, 1984.

BERG, R. Systematische untersunchungen über das verhalten der astes der aorta abdominalis bei Felis domestica. Anatomischer Anzeiger, Amsterdam, v. 110, p. 239-250, 1961.
BIASI, C. Aspectos comparativos entre os tipos de irrigação ventricular e do nó sinoatrial em coração de gato. 2009. 50 f. Dissertação (Mestrado em Anatomia dos Animais Domésticos e Silvestres) - Universidade de São Paulo, São Paulo. 2009.

BINVIGNAT, O.; OLAVE, E. Aspectos biométricos y morfológicos del pericardio fibroso y diafragma en el hombre. International Journal of Morphology, Temuco, v. 27, n. 3, p.841-847, 2009.

BONATELLI, M.; SANTOS, T. C.; PAPA, P.; AZARIAS, R. E. G. R.; MIGLINO, M. A.; PASSIPIÉRI, M.; MARTINS, D. S.; AMBRÓSIO, C. E.; OLIVEIRA, M. F. Descrição anatômica dos ramos da aorta abdominal do tamanduá bandeira (Myrmecophaga tridactyla). International Journal of Morphology, Temuco, v. 21, n. 1, p. 35-36, 2003.

CARVAlHO M. A. M.; MIGLINO, M. A.; DIDIO, L. J. A.; MELO, A. P. F. Artérias mesentéricas cranial e caudal em cutias (Dasyprocta aguti). Veterinária Notícias, Uberlândia, v. 2, p. $17-$ 24, 1999

CAVALCANTE FILHO, M. F.; MIGLINO, M. A.; MACHADO, G. V.; BEVILACQUA, E.; NEVES W. C. Estudo comparativo sobre o suprimento arterial do estômago do queixada (Tayassu pecari) e do cateto (Tayassu tajacu) [Linnaeus, 1789]. Brazilian Journal of Veterinary Research Animal Science, São Paulo, v. 35, n. 1, p. 20-24, 1998.

CULAU, P. O. V.; AZAMBUJA, R. C.; CAMPOS, R. Ramos colaterais viscerais da aorta abdominal em Myocastor coypus (nutria). Acta Scientiae Veterinariae, Porto Alegre, v. 36, n. 3, p. 241-247, 2008.

DYCE, K. M.; SACK, W. O.; WENSING, C. J. G. Tratado de Anatomia Veterinária. Rio de Janeiro: Editora Guanabara Koogan, 2004. 813 p.

DYCE, K. M.; WENSING, C. J. G.; SACK, W. O. Tratado de Anatomia Veterinária. Rio de Janeiro: Editora Guanabara Koogan, 2010. 856 p.

ENGE, I.; FLATMARK, A. Selective coeliac and hepatic artery angiography in normal dogs. Scandinavian Journal of Gastroenterology, Oslo, v. 7, p. 361-368, 1972.

EVANS, H. E. The heart and the arteries the dog. In: EVANS, H. E. (Ed.). Miller's anatomy of the dog. Philadelphya: Saunders, 1993. p. 586-681.

FIGUEIREDO, M. A.; SILVA, B. X.; CARDINOT, T. M.; BABINSKI, M. A; CHAGAS, M. A. Celiac artery in New Zealand rabbit: anatomical study of its origin and arrangement for experimental research and surgical practice. Pesquisa Veterinária Brasileira, Seropédica, v. 28, n. 5, p. 237-240, 2008.

GHOSHAL N. G. Coração e artérias do carnívoro. In: GETTY, R. (Ed.). Anatomia dos animais domésticos. 5 ed. Rio de Janeiro: Interamericana, 1986. p. 1497-1550.

GINTHER, O. J.; DIERSCHKE, D. J.; WALSH, S. W.; DEL CAMPO, C. H. Anatomy of arteries and veins of uterus and ovaries in Rhesus monkeys. Biology of Reproduction, New York, v. 11, p. 205-219, 1974.

HEBEL, R.; STROMBERG, M. W. Anatomy and Embriology of the laboratory rat. Verlag Worthsee: Biomed, 1986. p. 106-109.

HUAPAYA, J. A.; CHÁVEZ-TRUJILlO, K.; TRELLES, M.; CARBAJAL, R. D.; ESPADIN, R. F. Anatomic variations of the branches of the aortic arch in a Peruvian population. Medwave, San Francisco, v. 15, n. 6, e6194, 2015. 
INTERNATIONAL COMMITTEE ON VETERINARY GROSS ANATOMICAL NOMENCLATURE. Nomina Anatomica Veterinaria. Hannover: Editorial Committee, 2012. 160 p.

KENNEDY, H. N.; SMITH, A. W. An abnormal celiac artery in the dog. Veterinary Record, London, v. 10, p. 751, 1930.

KÖNIG, H. E.; LIEBICH, H. G. Anatomia dos animais domésticos. Texto e Atlas Colorido. Porto Alegre: Artmed, 2011. $788 \mathrm{p}$.

IUCN. The IUCN red list of threatened species. 2013. Disponível em: <http://www.iucnredlist.org/>. Acesso em: 7 jan. 2014.

LÓPEZ-GARCÍA, A.; FERNÁNDEZ, M. C.; DURÁN, A .C.; SANS-COMA, V.; FERNÁNDEZ, B. Quadricuspid aortic valves in Syrian hamsters and their formation according to current knowledge on valvulogenesis. Japanese Journal of Veterinary Research, Sapporo, v. 63, n. 1, p. 37-43, 2015.

LUDWIG, G.; AGUIAR, L. M.; ROCHA, V. J. Uma Avaliação da dieta, da área de vida e das estimativas populacionais de Cebus nigritus em um fragmento florestal no norte do estado do Paraná. Neotropical Primates, Belo Horizonte, v. 13, n. 3, p. 12-18, 2005.

MACEDO, B. C.; LIMA, A. R.; PEREIRA, L. C.; BRANCO, E. Descrição morfológica dos ramos colaterais da aorta abdominal do tamanduá-mirim (Tamandua tetradactyla). Biotemas, Florianópolis, v. 26, n. 1, p. 173-180, 2013.

MACHADO, G. V.; GONÇALVES, P. R.; PARIZZI, A.; SOUZA, J. R. Padrão de divisão e distribuição das artérias mesentéricas no ratão-do-banhado (Myocastor coypus - Rodentia: Mammalia). Biotemas, Florianópolis, v. 1, p. 59-63, 2006.

MACHADO, G. V.; SOUZA, J. R.; GONÇALVES, P. R.; PARIZZI, A.; DONIN, D. G. A artéria celíaca e seus ramos no ratão-dobanhado (Myocastor coypus - Rodentia: Mammalia). Biotemas, Florianópolis, v. 2, p. 41-54, 2002.

MACHADO, G. V.; ZIEMMAN, F. P. Comportamento anatômico das artérias mesentéricas no ouriço-cacheiro (Sphiggurus spp. Cuvier, 1825). In: EVINCI - EVENTO DE INICIAÇÃO CIENTÍFICA DA UFPR, 8, 2000, Curitiba. Anais... Curitiba: UFPR, 2000. p. 250.

McNAMARA, J. J.; FERGUSSON, D. J.; YAMASE, H.; SUEHIRO, G.; SOETER, J. R. Significant variations in distribution of the anterior descending coronary artery in primates. The Journal of Thoracic and Cardiovascular Surgery, Toronto, v. 65, n. 4, p. 639-642, 1973.

MICHLIK, K. M.; BIAZIK, A. K.; HENKLEWSKI, R. Z.; SZMIGIELSKA, M. A.; NICPOŃ, J. M.; PASŁAWSKA, U. Quadricuspid aortic valve and a ventricular septal defect in a horse. BMC Veterinary Research, London, v. 10, p. 142, 2014.

NAYAK, S. R.; PAI, M. M.; PRABHU, L. V.; D'COSTA, S.; SHETTY, P. Anatomical organization of aortic arch variations in the India: embryological basis and review. Jornal Vascular Brasileiro, São Paulo, v. 5, n. 2, p. 95-100, 2006.

NICKEL, R.; SCHUMMER, A.; SEIFERLE, E. The anatomy of the domestic animals. Berlin: Verlag Paul Parey, 1983. 610 p.

NIZA, M. M. R. E.; VILELA, C. L.; FERREIRA, A. J. A.; GONÇALVES, M. S.; PISCO, J. M. Irrigação arterial hepática em canídeo. Revista Portuguesa de Ciências Veterinárias, Lisboa, v. 98, n. 546, p. 69-76, 2003.
ORTALE, J. R.; MECIANO FILHO, J.; PACCOLA, A. M. F.; LEAL, J. G. P. G.; SCARANARI, C. A. Anatomia dos ramos lateral, diagonal e ântero superior no ventrículo esquerdo do coração humano. Revista Brasileira de Cirurgia Cardiovascular, São José do Rio Preto, v. 20, n. 2, p. 149-158, 2005.

PINHEIRO, G. S.; BRANCO, E.; PEREIRA, L. C.; LIMA, A. R. Morfologia, topografia e irrigação do coração do Tamandua tetradactyla. Arquivo Brasileiro de Medicina Veterinária e Zootecnia, Belo Horizonte, v. 66, n. 4, 2014. Doi: http://dx.doi. org/10.1590/1678-6844.

PINTO, M. P. E.; LIMA, A. R.; BRANCO, E. Morfologia, topografia e irrigação do coração do Saguinus niger. In: SEMINÁRIO ANUAL DE INICIAÇÃO CIENTÍFICA, 9, 2011, Belém. Anais... Belém: UFRA, 2011. Versão eletrônica.

REKHA, P.; SENTHILKUMAR, S. A study on branching pattern of human aortic arch and its variations in south indian population. Journal of Morphological Sciences, São Paulo, v. 30, n. 1, p. 1115, 2013.

SANTOS, A. L. Q; MENEZES, L. T.; BRITO, F. M. M; KAMINISHI, A. P. S.; LEONARDO, T. G.; PIMENTEL, T. L.; D'APARECIDA, N. S. Suprimento arterial para as glândulas adrenais de lobo marinho Arctocephalus australis (Zimmerman, 1783) (Pinnipedia, Otariidae). PUBVET, Londrina, v. 5, n. 11, p. 158, 2011.

SCHMIDT, S.; LOHSE, C. L.; SUTER, P. F. Branching pat-terns of the hepatic artery in the dog: arteriographic and anatomic study. American Journal of Veterinary Research, Schaumburg, v. 41, p. 1090-1097, 1980.

SEVERINO, R. S.; SILVA, F. O. C.; SANTOS, A. L. Q.; DRUMMOND, S. S.; SILVA, M.; ESTEVÃO, E. R. M. Irrigação das glândulas adrenais em gatos sem raça definida. In: SEMANA CIENTÍFICA DE MEDICINA VETERINÁRIA DA UNIVERSIDADE FEDERAL DE UBERLÂNDIA, 10, 1992, Uberlândia. Anais... Vol. 1, n. 1, Uberlândia: UFU, 1992. p.46-47. SILVA, F. O. C.; SEVERINO, R. S.; DRUMMOND, S. S.; MACHADO, G. V.; NOLASCO, R. N.; LIMA, E. M. M. Suprimento arterial para as glândulas adrenais em caprinos (Capra hircus) da raça Saanen. Arquivos de Ciências Veterinárias e Zoologia, Umuarama, v. 6, n. 1, p. 7-10, 2003.

SILVA, R. M. R. da; LIMA, A. R.; LACRETA Jr., A. C. C.; ISHIZAKI, M. N.; IMBELONI, A. A.; MUNIZ, J. A. P. C.; BRANCO, E. Descrição morfológica dos ramos colaterais viscerais da aorta abdominal do macaco-de-cheiro. Ciência Rural, Santa Maria, v. 41, n. 1, p. 94-100, 2011.

SLEIGHT, D. R.; THOMFORD, N. R. Gross anatomy of the blood supply and biliary drainage of the canine liver. Anatomical Record, Philadelphia, v. 166, p. 153-160, 1970.

SOARES, G. L.; OLIVEIRA, D.; BARALDI-ARTONI, S. M. Aspectos da anatomia do coração do avestruz. Ars Veterinaria, Jaboticabal, v. 26, n. 1, p. 38-42, 2010.

XAVIER-SILVA, B.; ROZA, M. S.; BABINSKI, M.A.; PALHANO, H. B.; SCHERER, P. O.; ABIDU-FIGUEIREDO, M. Ramos principais da artéria celíaca no gato doméstico: estudo aplicado à pesquisa experimental e à prática cirúrgica. Revista Brasileira de Ciência Veterinária, Niterói, v. 19, n. 3, p. 133-138, 2012. 